\title{
L'objet pauvre dans le théâtre contemporain
}

\author{
Jean-Luc Mattéoli
}

\section{(2) OpenEdition}

\section{Journals}

Édition électronique

URL : http://journals.openedition.org/imagesrevues/125

DOI : 10.4000/imagesrevues. 125

ISSN : 1778-3801

\section{Éditeur :}

Centre d'Histoire et Théorie des Arts, Groupe d'Anthropologie Historique de l'Occident Médiéval, Laboratoire d'Anthropologie Sociale, UMR 8210 Anthropologie et Histoire des Mondes Antiques

\section{Référence électronique}

Jean-Luc Mattéoli, «L'objet pauvre dans le théâtre contemporain », Images Re-vues [En ligne], 4 | 2007, document 4, mis en ligne le 01 janvier 2007, consulté le 30 janvier 2021. URL : http://

journals.openedition.org/imagesrevues/125; DOI : https://doi.org/10.4000/imagesrevues. 125

Ce document a été généré automatiquement le 30 janvier 2021.

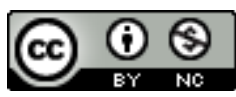

Images Re-vues est mise à disposition selon les termes de la Licence Creative Commons Attribution -

Pas d'Utilisation Commerciale 4.0 International. 


\section{L'objet pauvre dans le théâtre contemporain}

Jean-Luc Mattéoli

«L'objet a cessé d'être un accessoire de la scène, il est devenu le concurrent de l'acteur."

(Tadeusz Kantor, Leçons de Milan, 1990) « Faire du théâtre en rencontrant les restes. » (Michel Deutsch, « Dans la marge », 1999) 
1 Pour certains réformateurs de la scène du $\mathrm{XX}^{\mathrm{e}}$ siècle, l'objet n'est pas (ou plus) un accessoire : il est un des moyens avérés du travail mené avec l'acteur, au point de devenir un partenaire de ce dernier, voire son égal sur le plateau. Tadeusz Kantor en fournit l'illustration, et, d'une certaine manière, le fondement: sans doute parce qu'il était issu du milieu des Beaux-Arts,

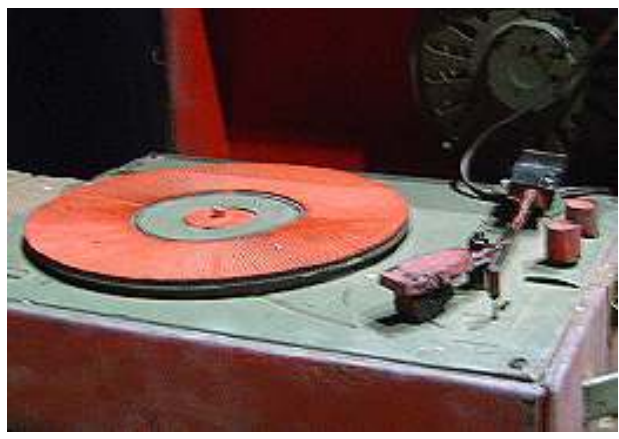
revendiquait nettement cette filiation plasticienne dans laquelle il inscrivait son théâtre, et que, à ses yeux, théâtre et arts plastiques constituaient donc les deux faces d'une même activité de création, Kantor a conféré aux objets des pouvoirs tout à fait particuliers, jusqu'à la provocation : "Sans eux », affirmait-il, "l'acteur n'existerait pas $»^{1}$. Sans objets, donc, pas de jeu, et, partant, pas de théâtre ? Si, au contraire d'autres, Kantor est peut-être l'un des rares metteurs en scène du XX $\mathrm{X}^{\mathrm{e}}$ siècle à n'avoir pas cherché à fonder une école d'acteurs, il a pourtant «fait école ». Et aujourd'hui, certains pourraient assurer, comme il l'affirmait dans un entretien avec Denis Bablet en 1972 : « Le problème de l'art, c'est toujours celui de l'objet ».

2 Cependant, il convient de prendre garde à deux points essentiels pour cette étude. L'objet que Kantor commence à utiliser en 1944 n'est pas l'accessoire ordinaire des théâtres : c'est un objet «vrai ». Cela signifie qu'il n'a pas été fabriqué pour les besoins de la (mise en) scène, mais qu'on l'a " arraché à la réalité de la vie » ${ }^{2}$ pour l'installer sur l'espace scénique, et l'y faire jouer. Cet objet vrai est aussi un objet "pauvre », en raison de son appartenance à « la réalité du rang le plus bas »: vieilles planches ou échafaudages de chantier, roue maculée de boue, chaises ordinaires, costumes élimés. Ce choix de l'objet extrait d'un réel sans grâce ni valeur est le signe, selon Kantor, d'une révolte née des convulsions de l'Histoire «contre toutes les saintetés prônées officiellement / contre tout ce qui est "confirmé" $»^{3}$ : en bref, contre l'idée de Beauté telle que l'ont véhiculée quatre siècles d'une histoire de l'art rendue désormais caduque par les cataclysmes historiques et économiques du $\mathrm{XX}^{\mathrm{e}}$ siècle.

3 Mais, d'autre part, les temps ont changé depuis 1944. Le basculement mémoriel provoqué dans les pays industrialisés par l'accélération du tempo économique, est sans doute ce qui a permis en Europe de l'Ouest la réception des spectacles du metteur en scène polonais à la fin des années 1970: la mémoire que contiennent les objets constitue l'un des moteurs de son travail, alors qu'elle devient un thème de société orientant les travaux de certains sociologues, ethnologues, historiens et artistes. L'accélération de l'histoire, perçue désormais par les masses, alimente une vision du passé rien moins que désireuse d'une nouvelle tabula rasa. Chute du mur de Berlin, effondrement de l'Union Soviétique : ce double naufrage - fin du monde bipolaire et effondrement des «Grands Récits » qui le soutenaient - a signé le retour de l'Histoire ; mais il a aussi renforcé la mémoire, en permettant à de nombreux occidentaux de visiter à l'Est des pays où coulait encore un temps "partout ailleurs révolu »" Le sentiment de la disparition et de la perte (objets, sites, monuments, paysages), engendré par des traumatismes mémoriels différents, nourrit désormais une inquiétude diffuse quant à l'avenir, en même temps qu'il charge certains objets à l'état 
de restes, au théâtre comme dans les arts plastiques, du pouvoir d'évoquer (au sens quasi étymologique) un passé en train de s'abolir.

4 La question, on le voit donc, est double: du point de vue de l'histoire du théatre, il s'agit de mesurer la manière dont l'héritage de Kantor, sur ce point particulier de l'objet, donne aujourd'hui lieu à des poétiques tout à fait distinctes de ce qui pouvait se passer à l'orée des années 1980, même si elles demeurent largement mémorielles; du point de vue de l'esthétique, il s'agit de saisir en quoi cette composante mémorielle de l'objet pauvre, aujourd'hui fortement marquée, contribue à modifier le régime ordinaire d'utilisation de l'objet au théâtre, et le tire vers des pratiques plus plasticiennes. Il s'agit donc de tenter de penser à nouveaux frais la place qu'occupe aujourd'hui l'objet dans ce travail théâtral particulier qui s'est effectué «dans la marge $»^{5}$ durant les trente dernières années, en n'oubliant pas, en bref, combien ses mutations dans le champ des arts plastiques ont affecté son statut sur la scène et comment Kantor fut l'artisan de cette articulation entre champs théâtral et plastique.

\section{Fondements}

5 Pour poser les bases de la réflexion, nous procèderons à un rapide examen des fonctionnements habituels de l'objet dans le théâtre régulier. Précisons que la place de l'acteur lui est évidemment liée. André Antoine, considéré souvent comme le premier metteur en scène français, n'écrivait-il pas, dans le Théâtre-Libre, en 1890, au moment où il plaide pour l'instauration de "décors exacts » sur les scènes, en lieu et place des toiles peintes : » Les expressions [de l'acteur] s'appuieront sur des accessoires familiers et réels, et un crayon retourné, une tasse renversée, seront aussi significatifs, d'un effet aussi intense sur l'esprit du spectateur que les exagérations grandiloquentes du théâtre romantique $»^{6}$ ? C'est dire combien l'objet, au moment où il est introduit en nombre sur les scènes naturalistes, est déjà dans un rapport de concurrence avec l'acteur (entendons l'acteur romantique, modèle du jeu académique en cette fin de siècle, et totalement inadapté au jeu naturaliste). Mais, près d'un siècle plus tard, l'usage dominant, même dans les tentatives les plus radicales de réforme, veut que l'objet soit inféodé à celui qui le manipule. Même pour Jerzy Grotowski, pourtant promoteur en 1964 d'un "théâtre pauvre ", c'est-à-dire rapporté à ses strictes et propres ressources, l'acteur, "par l'utilisation contrôlée de ses réactions, transforme le plancher en une mer, une table en confessionnal, un bout de fer en un partenaire animé, etc. $»^{7}$. Ainsi, même dans un théâtre dont l'essence est définie par sa "pauvreté ", l'acteur escamoterait les objets pour donner à voir d'autres «choses » en leur lieu et place, à la manière d'un illusionniste?

Dans "Problèmes du théâtre ", publié en 1940, Louis Jouvet sépare avec humour deux types de pièces: celles qui nécessitent pour être montées de réunir sur la scène un véritable "Mont de Piété » (en gros les théâtres romantiques et naturalistes), et, d'autre part, celles qui ne font appel qu'à quelques rares objets (en gros le théâtre classique); prenant évidemment parti pour ce dernier, il établit une correspondance entre le petit nombre d'objets présents sur scène et le succès de la pièce ${ }^{8}$. Pourquoi ? Peut-être parce que, dans une forme d'art fondée sur la présence de l'acteur dans les mêmes espace et temps que le public, les spectateurs sont nécessaires à l'effectuation scénique, et donc que, plus l'objet est rare sur le plateau, plus il mobilise leur imagination. Il suffira, pour s'en persuader, d'examiner successivement les trois modes 
majeurs d'insertion scénique de l'objet - en dehors du décor de type naturaliste, qui ne sera pas envisagé dans cette étude, l'objet y étant le signe de lui-même jusqu'à l'extrême limite.

7 Le premier cas est celui où l'objet, comme le décor, est invisible. Procédé ordinaire des théâtres de tréteaux et des acteurs de rue, dans lesquels l'itinérance et l'étroitesse des lieux théâtraux contraignent à ne transporter avec soi que l'essentiel, l'objet invisible est celui qui exige de l'acteur le plus d'énergie, puisque c'est de son jeu que dépend l'apparition de l'objet dans l'imagination du spectateur et donc la compréhension de ce qui se passe. Un exemple récent, tiré des Étourdis ${ }^{9}$ de Jérôme Deschamps et Macha Makeïeff, en fournit l'illustration : à un moment du spectacle, l'un des personnages (Patrice Thibaud) feint d'installer sur son bureau, successivement, trois machines à écrire. L'une est de taille normale ; la deuxième est japonaise, et donc miniaturisée ; la troisième est américaine, et donc énorme. Mais rien n'est visible : ni les machines, ni les cartons où elles sont emballées, ni leur chariot capricieux, ni les touches des claviers. Tout est en revanche esquissé dans l'espace par des gestes rapides du corps démultiplié de l'acteur, qui fait tourbillonner autour de lui objets et situations dans une complicité étroite avec le public. Litote au dernier degré, donc : il n'y a rien en scène que l'acteur, et pourtant le spectateur "voit» tous les objets avec lesquels celui-ci se débat. L'acteur-bateleur triomphe: son adresse, son énergie, son sens du trait juste, engendrent les rires et les applaudissements du public.

Le deuxième cas est celui où l'objet, effectivement présent sur scène, contribue à faire exister d'autres objets, absents, mais liés comme lui au même paradigme. Meyerhold en fut le promoteur; critiquant le naturalisme de Stanislavski, il raillait: "[Le théâtre naturaliste] a voulu que, sur scène, tout soit "comme dans la vie", et il s'est transformé de ce fait en une boutique d'objets de musée ${ }^{10}$. A l'opposé, donc, du "décor exact » des naturalistes, l'élève de Stanislavski défend l'idée que l'art du théâtre se fonde sur la convention, c'est-à-dire la manière dont le metteur en scène "place[r] l'imagination [du spectateur] sur la bonne voie en lui laissant le dernier mot $»^{11}$. Il s'agit donc de suggérer ce qui est absent du plateau sans encombrer ce dernier : un objet sert d'indice, le reste du décor étant simplement induit, dans l'imagination du spectateur, par ce qui lui est présenté. Ainsi, une seule table à café installée sur scène, aisément identifiable à son piètement et à son plateau rond (et, accessoirement, à la chaise elle aussi typique qui l'accompagne), suffira par exemple à suggérer la salle entière du café et son atmosphère, tels que la pièce les suppose : les autres tables, le comptoir, les miroirs aux murs, etc., sont invisibles, mais chaque spectateur (re)construit l'ensemble de la scénographie dans sa tête, grâce au texte, à la présence d'un serveur en habit, etc. Meyerhold nommait cette forme de réalisme " réalisme astringent ", les signes destinés à donner l'illusion d'un lieu étant en effet " resserrés ", réduits en somme à l'essentiel. Ce type de scénographie réaliste, laissant une fois de plus la part belle à l'acteur, est devenu courant sur les scènes du XXe siècle, et Brecht en reprendra la leçon : choisir des indices pour laisser le reste à imaginer au spectateur, quitte à paraître " parcimonieux », voire (encore une fois) " pauvre ${ }^{12}$.

Le troisième et dernier cas est celui qui est sans doute le plus familier, mais aussi le plus intéressant pour cette étude, car il est fondé sur la métaphore. Un objet quelconque, manipulé par un acteur, change de nature pour un instant par la grâce du jeu: le chapeau d'Arlequin, rentré dans le col du vêtement, devient la serviette d'un repas improvisé, et sa batte, glissée entre les jambes, un phallus surdimensionné quand passe 
une jolie servante. Ainsi, on peut représenter un grand nombre d'objets à l'aide d'un seul, à condition qu'il soit manipulé par le comédien de manière à se placer dans une configuration de gestes aisément identifiable par le spectateur: effectuer le mouvement du lanceur de poignard, bras levé au-dessus de la tête, jambe en avant, suffit à transformer effectivement, dans l'imagination du regardeur, l'objet qu'on tient à la main (quel qu'il soit) en une arme blanche. C'est en effet l'ensemble des gestes et mimiques développés autour du chapeau-serviette d'Arlequin qui construit le contexte dans lequel l'objet métaphorisé s'insère. Car telle est la règle de fonctionnement de l'objet dans le théâtre régulier: il s'efface dans le tremblé de la métaphore (le spectateur ne " voit " plus le chapeau), et fait apparaitre l'objet absent (une serviette), exerçant de la sorte ce «muscle » qu'est l'imagination du spectateur, et laissant ainsi une place à ce dernier dans le processus de la représentation. Illustration patente de la compétence du metteur en scène ou de l'acteur, l'objet, transformé par la métonymie ou la métaphore, peut se trouver ainsi enfermé dans une fonction ancillaire (servir en s'effaçant) contre laquelle Kantor s'est érigé.

Fig.1.

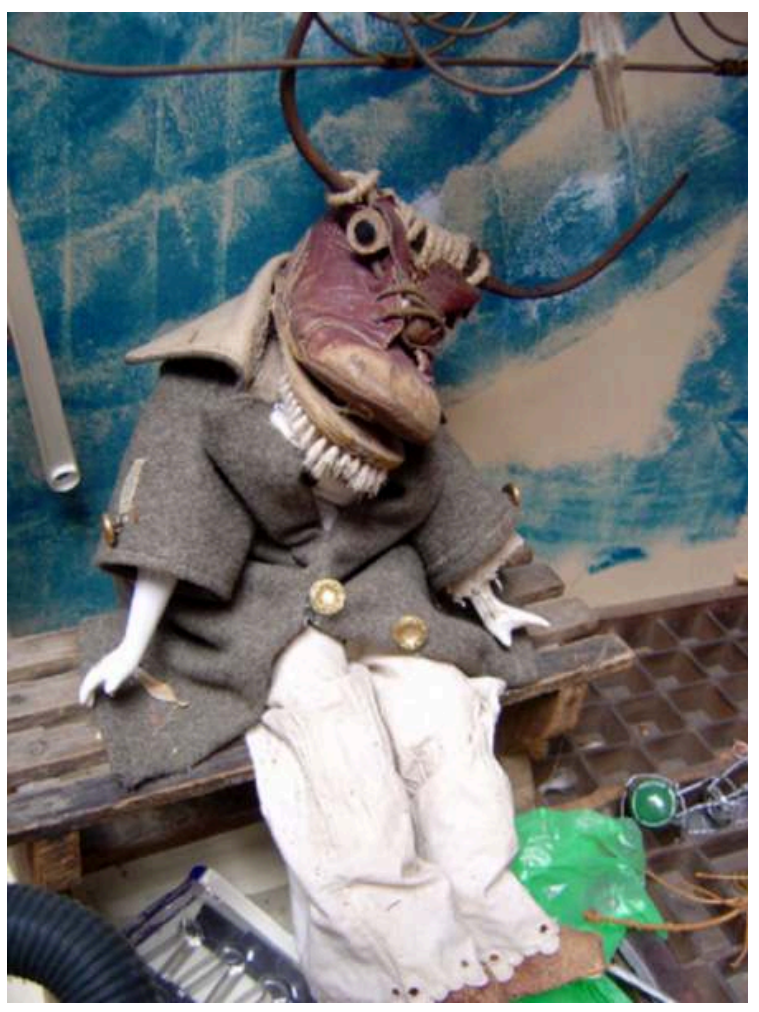

Deux objets (une brosse, une empeigne de chaussure) métaphorisés en un visage taurin. Turak Théâtre, atelier de la rue Perrod (Lyon).

(C) J.L. Mattéoli

L'objet pauvre peut lui aussi relever des types de fonctionnement métonymique ou métaphorique. Rien, a priori, ne l'en empêche, à l'instar de n'importe quel autre objet sur scène. Cependant, à observer ce qui se produit aujourd'hui dans le théâtre d'objet ou dans certains théâtres d'acteurs, on ne peut que constater la manière dont il induit également des manières de faire assez différentes, tout en étant toujours susceptible des fonctionnements répertoriés ci-dessus (Fig. 1). 
11 Il n'est pas, redisons-le, un accessoire fabriqué ou inventé par un accessoiriste. Il n'a d'ailleurs plus rien de l'accessoire, si l'on définit ainsi l'objet qui sert à l'acteur à asseoir une dimension de son personnage, et qui, portant bien son nom, joue néanmoins un rôle secondaire dans cette construction. Kantor le dirait dans ce cas « servile » : "Chez moi, il n'y a pas d'accessoires. Les accessoires sont des choses terribles, "utilisables" seulement pour faire quelque chose. Ce ne sont pas des objets, ils ne recèlent aucun danger. $\mathrm{Ce}$ sont des attrapes ${ }^{13}$. L'objet se distinguerait ainsi de l'accessoire en cela qu'il présenterait « un danger »? Bien évidemment, le mot n'est pas à prendre au pied de la lettre, mais il suggère cependant que l'objet, au contraire de l'accessoire dont le faisceau des significations et des réactions physiques est a priori maîtrisé, peut continuer à jouer. «Jouer ", c'est-à-dire «faire des propositions » et répondre à celles de l'acteur, voire le surprendre car tout « jeu » (comme entre deux pièces mécaniques) suppose un décalage. De même qu'une oeuvre d'art ou une machine (Kantor compare les objets de ses spectacles aux premières et introduit sur scène les secondes), l'objet vrai a une existence propre, et à ce titre, il agit. Point n'est besoin de le manipuler pour cela : sa simple présence immobile suffit.

D'autres questions surgissent cependant de ces premiers éclaircissements : alors qu'il est devenu désormais égal et partenaire de l'acteur, en quoi sa nature d'objet « arraché à la réalité » peut-elle contribuer à modifier ainsi les poétiques de l'objet théâtral ? D'autre part, ce passage de la transformation (qui escamote l'objet au profit de l'illusion) à l'exposition (qui l'exhibe au profit de la distance), intervenu en une trentaine d'années entre 1975 et aujourd'hui, pourrait-il constituer une des mutations remarquables de l'objet de théâtre en ce début de siècle?

\section{Un peu d'histoire}

La présence de l'objet arraché au réel semble donc modifier le régime de fonctionnement de l'objet sur la scène moderne, et lui conférer un nouveau statut: d'accessoire, il devient acteur. Mais qu'est-ce, d'abord, qu'un objet récupéré ?

Dans la plupart des cas, c'est un objet qui est sauvé de la poubelle, c'est-à-dire de la disparition. C'est, comme l'écrit Kantor :

Un objet misérable, PAUVRE, incapable de servir dans

la vie, bon à jeter aux ordures.

Débarrassé de sa fonction vitale, protectrice,

nu désintéressé, artistique!

Appelant la pitié et L'ÉMOTION !

C'était un objet complètement différent de l'autre,

Une roue boueuse de charrette,

Une planche pourrie,

Un échafaudage de maçon barbouillé de chaux,

Un horrible haut-parleur hurlant les communiqués de guerre...

sans voix...

Une chaise de cuisine ${ }^{14}$. 
Fig.2.



Le tourne-disque, désormais obsolète, participe de la temporalité complexe de la scène. Théâtre de la Licorne, Bestiaire forain création en 2001 au Channel (Calais).

Cliché : J.-L. Mattéoli

Il peut avoir été jeté (le cas est fréquent dans les sociétés modernes fondées sur l'obsolescence) parce qu'il était dépassé technologiquement, ou démodé (Fig. 2). Mais, le plus souvent, c'est parce qu'il est hors d'usage. Bosselé par un choc malencontreux, éraflé, voire franchement cassé (en plusieurs morceaux), souillé et poussiéreux, l'objet récupéré est inapte à remplir l'office pour lequel il a été conçu. De la sorte, étrangéisé par son caractère fragmentaire ou dépassé, comme par les formes nouvelles que les coups ou la rouille ont dessiné sur son enveloppe, l'objet, apparemment désaffecté, est prêt pour d'autres usages que ceux pour lesquels il a été conçu, comme si le bris et l'usure avaient libéré ses potentialités artistiques.

16 Cette pratique de la récupération est-elle nouvelle? Elle a grosso modo l'âge de la modernité, et, en ce qui concerne le théâtre, certaines de ses origines sont aisées à identifier. Sans préjudice de cet usage courant qui consiste à annexer au plateau, faute de mieux, les objets personnels des comédiens, les scénographies de Bertolt Brecht représentent un moment relativement premier de cette pratique d'appropriation: vraisemblablement à l'automne 1936, il évoque déjà ces objets « d'occasion » - un bâti de porte, par exemple, pris sur un " chantier de démolition » - dont il recommande de s'entourer pour mettre en place une architecture scénique susceptible de rendre insolite aux spectateurs des lieux ou des usages par trop connus ${ }^{15}$. Refusant l'illusion intégrale, la scénographie brechtienne met à distance le regard du spectateur en lui fournissant une vue incomplète, quoique toujours réaliste, du monde qui l'entoure. Elle lui rappelle ainsi constamment qu'il se trouve au théâtre et favorise l'historicisation des événements présentés sur le plateau. Kantor constitue une autre origine : en juin 1944, dans Varsovie encore occupée par l'armée allemande, il a recours aux premiers 
objets "pauvres" pour présenter à un public clandestin Le Retour d'Ulysse . "Nous n'avions assez de force ", écrit-il en 1986, "que pour attraper ce qui était sous la main , l'objet réel, et le proclamer oeuvre d'art ! $»^{16}$. Cette fois cependant, il s'agit non pas tant $\mathrm{d}$ 'historiciser que de toucher, non d'exercer la raison mais de faire couler les larmes, et de « créer un théâtre qui aurait une puissance d'action primitive, bouleversante ! $»^{17}$. 1936, 1944 : les dates sont proches, à la fois entre elles (à peine une dizaine d'années séparent les deux "découvertes »), mais aussi des cataclysmes que représentèrent les deux guerres mondiales. Le plasticien Kurt Schwitters éclaire dès 1919 le lien qu'entretiennent les objets pauvres et la destruction : «Comme le pays était ruiné, par économie, je pris ce qui me tombait sous la main. On peut crier aussi avec des ordures et c'est ce que je fis, en les collant et les clouant ensemble ${ }^{18}$. Ainsi se trouveraient rapprochées les guerres mondiales, catastrophes pourvoyeuses de débris, de restes, et certaines pratiques artistiques (préexistantes pour certaines) fondées sur la récupération d'objets : quand des systèmes de valeurs s'effondrent devant la brutalité ou la résistance du réel, les objets rebutés semblent alors être introduits plus facilement dans le champ des pratiques artistiques. Avec les Surréalistes, un peu plus tard, la composante temporelle (et mémorielle) infléchit la recherche de l'objet : André Breton le choisit "démodé ", "énigmatique " ${ }^{19}$, et donc suffisamment étrange pour corriger la règle d'airain de l'usage, dans le but de faire pièce à l'envahissement du monde par les objets communs.

Une dernière étape pourrait être constituée par la pensée de Jerzy Grotowski, à la fin des années 1960 en Europe, qui répand les principes du "Théâtre pauvre ", en réaction contre les menaces que fait peser sur le théâtre la concurrence du film et de la télévision : cette menace oblige à délimiter, en une démarche essentialiste, le champ propre de l'art théâtral, qui est celui d'un anti-technicisme foncier. En 1980, Anne Ubersfeld fait par exemple du travail métaphorique avec l'objet (à mains nues) une «recette » grotowskienne. Distinguons une dernière fois cette approche du théâtre et de l'objet (quoique "pauvre ", elle demeure essentiellement illusionniste et fondée sur l'effacement de l'objet) de celle de Tadeusz Kantor qui promeut, lui, « l'objet pauvre ", celui qui ne s'efface pas, mais qui, au contraire, sauvé de la disparition, revient, insistant, du rivage des morts pour exister une deuxième fois, sur la scène. L'acteur kantorien ne l'escamote pas: au contraire, il doit «tout faire pour que l'objet soit visible ».

Cette pauvreté de l'objet (qu'on pourrait définir comme un mélange entre l'émotion éprouvée devant le témoin d'un monde disparu, et l'indifférence ordinaire suscitée par ce qui est commun), comment peut-elle exercer son influence sur les poétiques de l'objet théâtral ? Ce dernier, hier encore, cessait sur scène d'être lui-même, c'est-à-dire une seule chose, " pour devenir ce que veulent à un moment déterminé du spectacle, à la fois l'acteur et l'action dramatique $\aleph^{20}$ : en quoi le poids de mémoire qu'il contient peut-il brouiller ce rapport d'obéissance, voire de servilité, qu'il entretient avec le comédien, et faire, en quelque sorte, qu'il retrouve son unicité ? La première réponse, immédiate, est à chercher du côté de cette vie que les créateurs y pressentent. L'objet est en effet un "rescapé » (pour Macha Makeïeff), "appelant la pitié et l'émotion » (pour Kantor) : la personnification signifie sans ambiguïté que quelque chose d'humain s'est déposé en lui. Est-ce sa condition de rebut qui ainsi le désigne à la pitié ? Est-ce donc parce qu'on l'a "abandonné », comme le dit Nicolas Diaz ${ }^{21}$ - ce verbe suggérant le refus de s'occuper de quelqu'un alors que c'eût été notre devoir? Il est évident que 
l'adjectif «pauvre » est à double sens : l'objet est pauvre parce que, quotidien, il est dépourvu des traits qui pourraient le hisser à hauteur d'art (pour le sens commun, l'art exclut la trivialité du quotidien), mais il l'est aussi parce qu'on le plaint d'en être réduit à cette extrémité que constituent la rue, la poubelle, l'ordure. L'objet appelle la pitié car, dans la compagnie des hommes, il s'est chargé d'humanité, et que, son service achevé, il est mis au rancart comme un domestique désormais trop vieux ou fatigué. Mais, lesté d'une mémoire dont il a été le réceptacle muet durant un temps plus ou moins long, il n'est plus ce simple objet sur lequel son origine et son existence uniquement matérielles interdiraient de s'émouvoir, et qu'on jetterait sans état d'âme. L'objet pauvre est un réservoir à mémoire, et son apparition au théâtre dans le milieu des années 1970 n'a donc rien de fortuit.

C'est en effet le moment que certains historiens identifient comme le «basculement mémoriel des années 1970 »: "une société saisie par la croissance et brusquement coupée de ses racines $»^{22}$, écrit l'historien Antoine Prost, s'interroge sur les chemins qui l'ont conduite à ce point de rupture, et sur les moyens de ne pas perdre tout à fait le contact avec un monde, pourtant, dont on parait à jamais coupés. Dix ans plus tard, en 1988, Georges Banu repère cette mutation importante au théâtre, dont il lit les effets jusque dans le théâtre régulier: "De l'histoire vers la mémoire ", écrit-il, "c'est le chemin suivi $\aleph^{23}$. L'objet mémoriel, ainsi, serait vivant dans la mesure où il est le réceptacle d'histoires, d'affects, de souvenirs qu'y a déposé l'existence de son propriétaire, dans un monde maintenant disparu, et mon hypothèse est que c'est en raison de cette vie mémorielle qu'il résiste à la manipulation cherchant à le rendre soit vivant, soit autre, bref à le priver de lui-même.

Il y a en effet deux types de manipulation. La première est marionnettique, et « vise à "donner la vie" ${ }^{24}$ à l'objet; la seconde, la plus courante nous l'avons vu, consiste à le métaphoriser. Or l'objet pauvre n'est-il pas déjà vivant? Certes, il l'est, mais, comme incomplet, faisant entendre de " confuses paroles ", l'objet abandonné puis récupéré en appelle à l'imagination du metteur en scène et des acteurs, ces archéologues qui l'ont extrait du cours ordinaire des jours, pour en construire la ou les histoires ${ }^{25}$. L'animer, ce serait le priver de cette vie et de cette parole propre qu'il recèle ; le métaphoriser, ce serait le rendre invisible, en brouiller la "présence ", voire l'escamoter, et avec elle tout ce et ceux que l'objet, en quelque sorte, contient.

\section{La fin de la métaphore}

On n'en finirait aujourd'hui pas de repérer, dans différents champs artistiques, les signes d'une tendance certaine à la dé-métaphorisation, à l'œuvre depuis le milieu des années 1970. Au cœur de la modernité, Kafka, déjà, disait sa défiance pour une figure de style qui transforme le monde au lieu d'en rendre simplement compte dans son absurde complexité. La métaphore, trope inoculée au langage par une conception de la poésie désormais éculée, est en effet, étymologiquement, un transport: elle évade donc du monde tel qu'il est, substituant par exemple à l'ordinaire poire le «long fruit d'or » déjà raillé en son temps par Victor Hugo. Certains poètes s'écartent donc de cette figure, cherchant davantage à rapprocher la poésie de la « biscotte sans beurre » dont parle, avec un peu de provocation, Emmanuel Hocquart ${ }^{26}$. En dégraissant le langage du lyrisme (voire de la boursouflure, et, à coup sûr, du mensonge), les poètes d'aujourd'hui sont à ses yeux les « ingénieurs-grammairiens du langage ordinaire ${ }^{27}$ : ils cherchent, 
non à transformer, mais à accueillir, sur la scène de l'espace littéraire, "le même sorti de son contexte $\aleph^{28}$. Mutatis mutandis, il serait aisé de découvrir, aujourd'hui, dans l'utilisation qui est faite de l'objet pauvre au théâtre, une même tendance à éviter la métaphorisation, plus ou moins affirmée suivant les compagnies. Cette désaffection pour la métaphore s'accompagne d'une défiance, plus ou moins affirmée, pour la manipulation d'une façon générale.

Dans le théâtre d'objet par exemple, paradoxalement, l'objet n'est pas toujours manipulé. Le comédien de Catalogue de voyage ${ }^{29}$, du Théâtre de Cuisine, met en scène à la fin du spectacle deux objets en plâtre peint : une tête de panthère (un cache-pot), et une autre de berger allemand (ceux dont on orne parfois les dessus de cheminée ou de télévision). Bien loin de les manipuler pour donner l'illusion qu'ils sont vivants, le comédien se tient derrière eux, se déplaçant de l'un à l'autre lorsqu'il s'agit de leur donner la parole. Il n'y a de vie que celle que le spectateur projette lui-même. Au-delà de la marionnette, l'objet peut aussi être, comme le dit Christian Carrignon ${ }^{30}$, un "lieu » avant tout. Dans Vingt minutes sous les mers ${ }^{31}$, du même Théâtre de Cuisine, Katy Deville, grâce à un simple objet, crée deux lieux : plongeant une minuscule poupée dans un aquarium, elle la fait évoluer en bruitant ses soupirs d'aise ; le petit objet vient-il à plonger, la comédienne gonfle les joues, écarquille les yeux, bruite l'air qui sort de sa bouche, et le spectateur a l'impression d'être sous l'eau avec la poupée. Comme au cinéma, deux plans successifs, dirait-on: en fait, ils sont simultanés, puisque le spectateur saisit du même coup d'œil la figurine dans l'aquarium ou la comédienne qui les surplombe. Mais l'aquarium reste aquarium, et la poupée demeure poupée : c'est la comédienne, au rebours de la discrétion légendaire du marionnettiste, qui se transforme, tantôt manipulatrice, tantôt personnage. L'objet, lui, résiste. 
Fig.3.

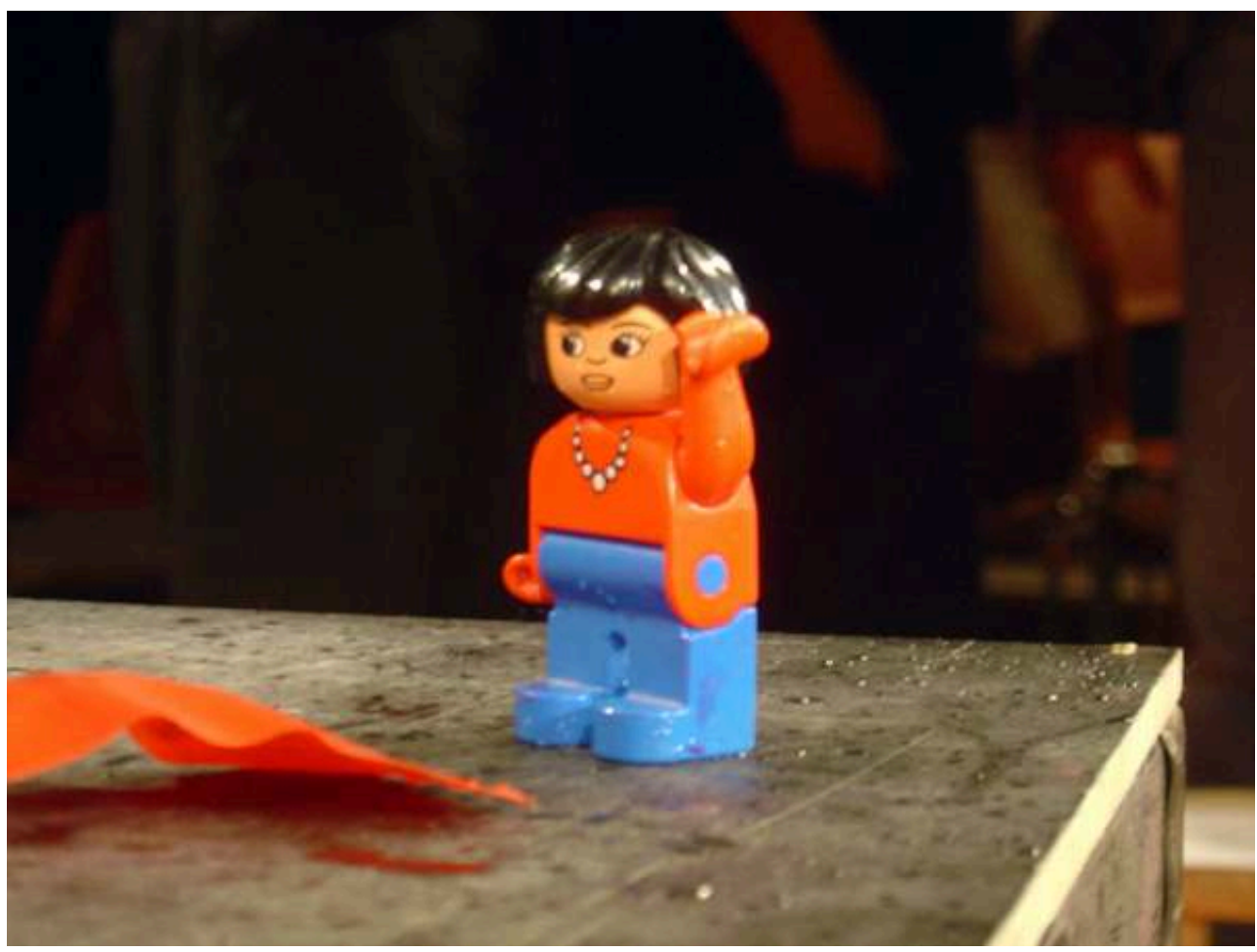

Un objet jouet : la figurine Playmobil au visage figé. Théâtre Manarf, Paris-Bonjour ; réaction en 1979 à la MJC d'Avrilié.

Cliché : J.-L. Mattéoli

Le cas que fait le théâtre d'objet des jouets, et notamment des figurines, mérite d'ailleurs qu'on s'y attarde un instant: type même de l'objet ordinaire, la figurine de Barbie, de Ken, ou d'Action Joe résiste par définition à la transformation (Fig. 3). D'abord parce que les traits du visage et les articulations du corps sont souvent figées par le plastique en des expressions ou des postures définitives, alors que la marionnette se définit justement par les articulations qui lui permettent le mouvement ; de surcroît, alors que les traits du visage des figurines sont précisément peints en des expressions convenues (généralement souriantes), la marionnette gagne évidemment à être la plus fruste possible, afin de ménager à la lumière et à la manipulation de multiples occasions de faire changer son apparence, et donc les émotions qu'elle procurera au spectateur ${ }^{32}$. L'objet figé, lui, fût-il anthropomorphe, n'est pas susceptible de tels mystères, et le comédien ne cherche pas à y fait croire le spectateur. 
Fig.4.

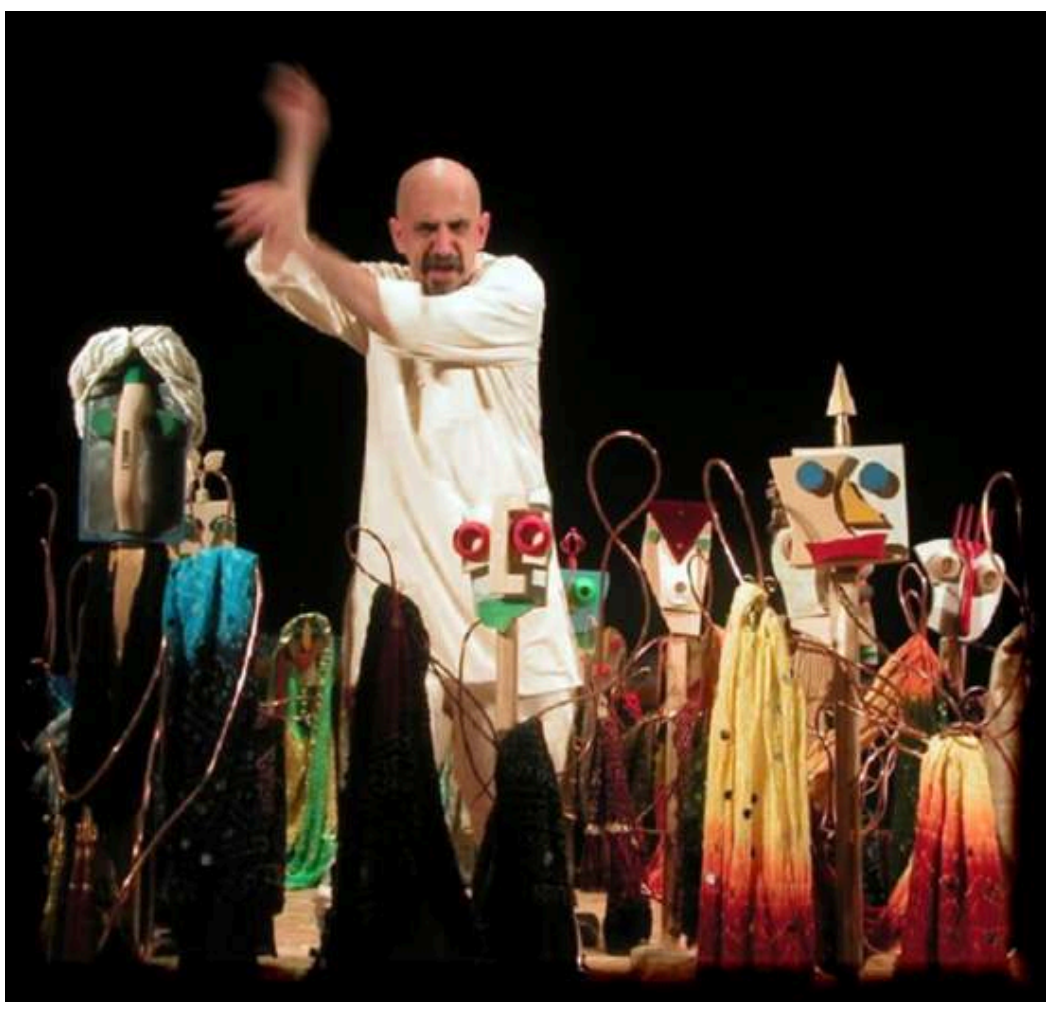

Les grandes marionnettes; Théâtre de l'Arc-en-Terre, Mahabharata

Fig.5.

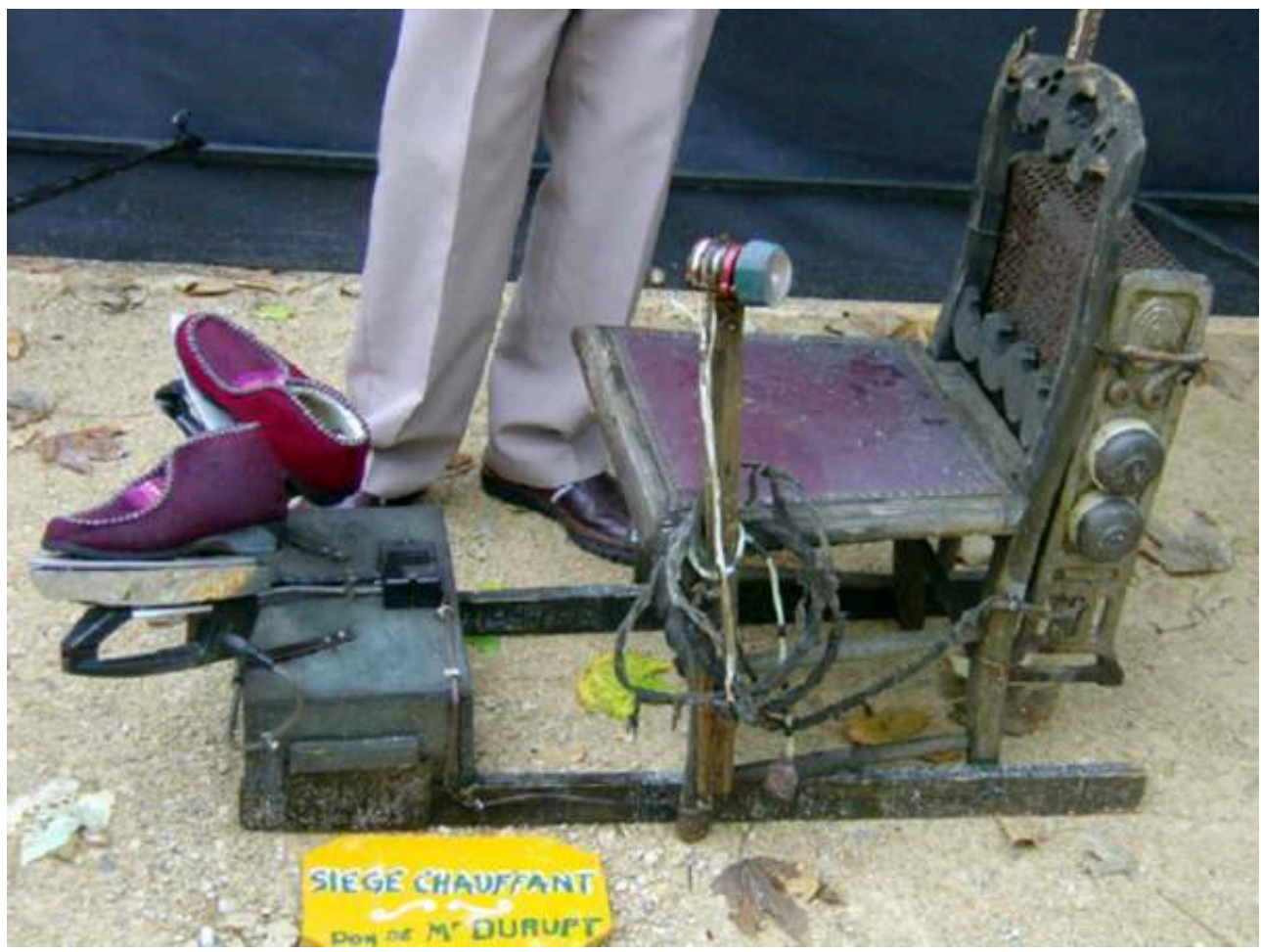

Les objets résistent, même réunis dans une machine. Office des Phabricants d'Univers Singuliers, Le Petit Répertoire. Création par couches successives de 2001 à 2004.

Cliché : J.-L. Mattéoli 
e pris dans un mouvement de métaphorisation, l'objet pauvre résiste encore, difficilement soluble dans l'ensemble auquel il participe. Les grandes marionnettes fabriquées par Enrico Baj pour Massimo Schuster, de la compagnie de L'Arc-en-Terre, en constituent des exemples frappants : constitués d'objets achetés en grande surface de bricolage, les traits de leur visage sont figurés par des râteaux ou des pelles de jardinage, des anneaux de tringle à rideau, etc. que rien ne vient fondre dans un tout, et que leurs couleurs contrastées achèvent même de rendre très visibles et identifiables (Fig. 4). Il en va de même dans les réalisations des artistes imaginaires de la compagnie OPUS (Fig. 5). Le bricolage, très apparent, signale la présence de l'homme dans l'objet offert aux regards, au contraire de beaucoup d'objets contemporains, parfaitement carrossés, sans suture ni assemblage apparent, aussi lisses que le corps d'un mannequin, comme en provenance directe du futur, selon la croyance technologique en la fécondité de cette portion du temps: le caractère magique de ces objets se nourrit aussi de leur aspect lisse, comme étranger à la main de l'homme.

\section{Bricolage, rafistolage}

26 À cette résistance de l'objet pauvre, il y a, corollaire de son poids de mémoire, une autre raison : il est le produit d'un bricolage. Travailler avec des objets récupérés, c'est en effet s'inscrire dans un cadre que Claude Lévi-Strauss a défini dans La Pensée sauvage : selon lui, tout bricolage serait rétrospectif, se fondant sur « une collection de résidus d'ouvrages humains $\aleph^{33}$. L'objet né du bricolage est donc constitué d'éléments hétérogènes, conjugués au passé, et assemblés avec plus ou moins de solidité et de bonheur: mais, par cela même qu'il est imparfait, il s'avoue nettement d'origine humaine. Certaines compagnies, comme le Théâtre de La Licorne, vont d'ailleurs jusqu'à fabriquer elles-mêmes les objets dont elles ont besoin pour gagner sur le temps ordinairement consacré à leur récupération, quitte à les patiner ensuite pour leur donner l'air ancien: l'électronique, l'informatique nécessaires à l'évolution des animaux-machines du Bestaire forain ${ }^{34}$ sont alors camouflées, mais la mécanique, signe du bricolage et de l'artisanat, est, elle, exhibée . Ainsi bricoleur et objet pauvre sont-ils étroitement liés. Dans le bricolage, enfin, c'est l'objet qui détermine le projet du bricoleur, lequel assemble en fonction de ce qu'il a «sous la main » (comme le disaient Kantor et Schwitters) : outils et matériaux ne sont pas toujours les bons, mais le résultat, ainsi, dépendra du bricoleur autant que des objets qui l'entourent.

Exemple, donc, d'un fonctionnement technique dans lequel le matériau a voix au chapitre, le bricolage est l'exact pendant du progrès technologique accéléré des sociétés modernes : le premier récupère (donc détourne et recycle) ce que le dernier condamne comme obsolète, et, ce faisant, lutte contre la disparition. Il n'y a donc rien d'étonnant à ce que l'objet ne soit pas escamoté par le jeu, transformé ou manipulé au service de l'illusion: on éprouve en effet aujourd'hui quelque difficulté à réduire au silence de la domesticité celui qui, revenu vivant d'entre les morts, est chargé d'un poids de mémoire qui suffit à entraîner le respect et à provoquer l'imagination. Assemblé en une machine, l'objet pauvre constitue de surcroît une critique en acte du modernisme : la bricole s'y moque en effet de la technologie. Dans C'est magnifique, de la compagnie Deschamps \& Makeïeff ${ }^{35}$, quelques planches, des lessiveuses en métal et des soucoupes de plastique coloré, assemblées en un improbable circuit servi par les comédiens, constituent en un tour de mains une machine bruyante et compliquée dont 
la seule fonction est de collecter, à l'arrivée, quatre mètres plus loin, les soucoupes qu'on y avait engagées, dans le même état et dans le même désordre (ce retour au point de départ est d'ailleurs souvent le fondement du burlesque tel que le conçoit cette compagnie). Pendant ce temps, les comédiens sont devenus les serviteurs de la machine inutile : réifiés, automatiques, et précis, avec cependant l'étincelle de folie des clowns.

Fig.6.

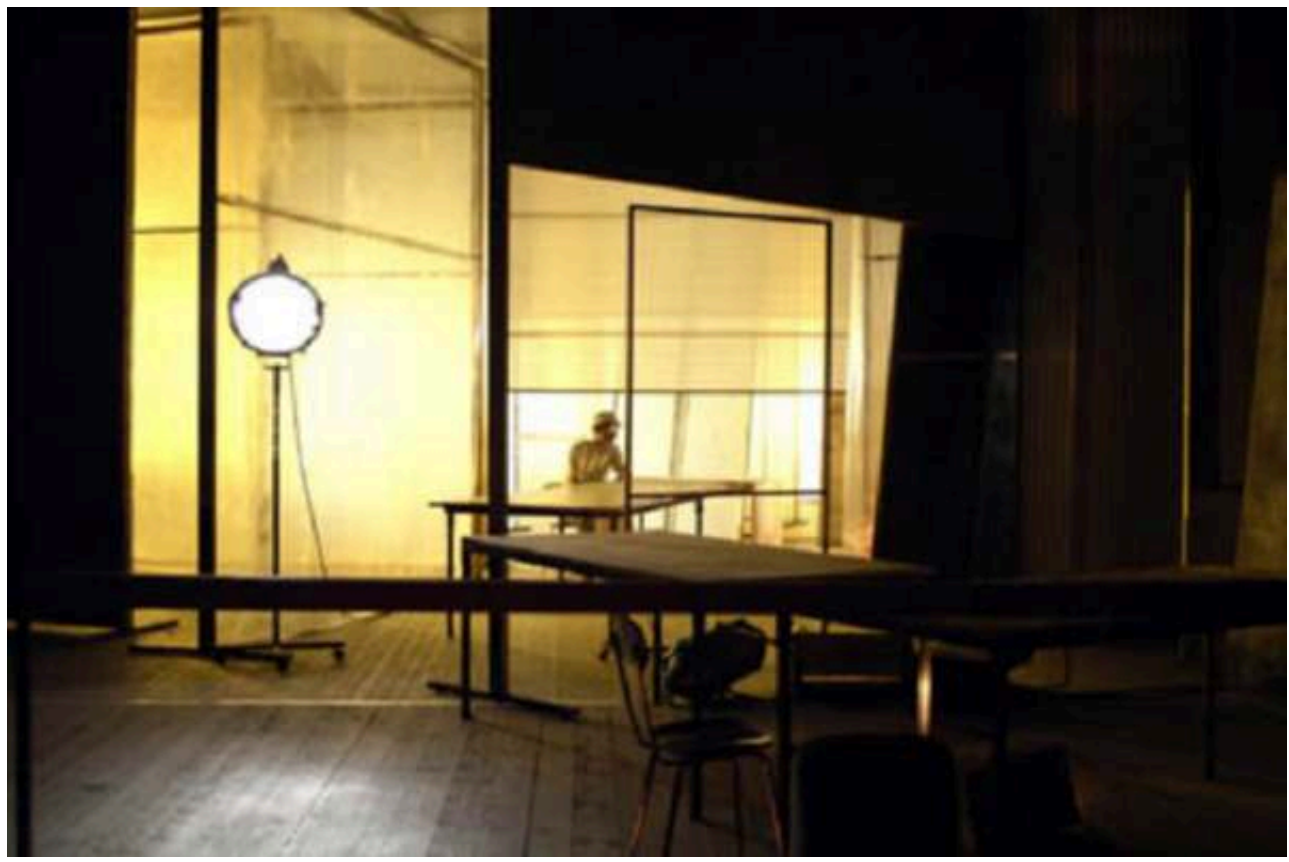

Tables et chaises de collectivité, éclairages de chantier, panneaux tendus de papier cadeau ou kraft: chaque composante de la cérémonie théâtrale est dégradée en objets ordinaires. Théâtre du Radeau, Coda. Création au Mans en octobre 2004.

Cliché : D. Grappe

L'objet pauvre tel qu'il fonctionne au Théâtre du Radeau met le spectateur face à une ultime sorte de bricolage, où l'on fait là aussi avec ce qu'on a sous la main, mais ce qu'on a sous la main est tellement pauvre... Le travail que mène cette compagnie depuis plus de vingt ans est exemplaire de cette prise en compte du mot fameux d'Adorno selon lequel, après Auschwitz, toute forme de poésie serait à bannir. Les deux guerres mondiales, avec leur cortège d'horreurs, ont constitué en effet des césures indéniables sur de multiples plans, nous l'avons évoqué plus haut. Dans le domaine artistique, on l'a vu pour Schwitters, Brecht ou Kantor, elles portent deux coups de boutoir définitifs à l'esthétique symboliste, pétrie de visions gréco-latines ou germaniques, arc-boutée sur la notion éternelle du Beau, et déjà mise à mal dès la fin du XIX ${ }^{\mathrm{e}}$ siècle. Pour le théâtre du Radeau, plus rien ne peut donc être comme avant, aussi joue-t-il dans un décor de restes. C'est avec ces restes - des tables de collectivités en formica par exemple - qu'on fera une scène ; des néons fixés sur ces mêmes tables seront la rampe ; les vêtements au bord du déguisement, mal enfilés, violemment disparates sont des restes de costumes; restes des toiles peintes, ces grands panneaux tendus de papier cadeau ou de papier kraft, que les comédiens ne cessent de déplacer dans l'espace; restes de la salle, cette "tente » démontable sans confort, dans laquelle comédiens et spectateurs sont installés au même niveau ; restes de lumières, enfin, ces projecteurs de chantiers posés à terre, dont les câbles traînent sur le plateau (Fig. 6). 
Au milieu de cet espace où les composantes du spectacle théâtral sont dégradées dans des objets pauvres, pour certains hâtivement fabriqués, il est d'autres objets, étrangers, eux, au théâtre : absolument ordinaires, ils peuvent être décoratifs (un lampadaire de salon, un grand cerf en plastique, des instruments de musique) ou fonctionnels (un ventilateur, un électrophone, un projecteur à diapositives). Mais, dans quelque catégorie qu'ils s'inscrivent, ils sont apportés, déplacés, posés (exposés), remportés par les comédiens sans que leur jeu en soit affecté, ou que jamais leur jeu ne les affecte (notamment par une quelconque métaphorisation). L'objet est simplement là, réduit à sa fonctionnalité têtue, en-deçà du signe théâtral ordinaire, puisque totalement isolé d'autres signes (ou constellations de signes) avec lesquels tisser une image de lieu référentiel possible. Ainsi le lampadaire ne signe-t-il pour aucune illusion d'appartement, et les costumes pour aucun personnage identifiable dans la durée (tous les acteurs masculins sont par exemple habillés de la même façon depuis La Bataille $d u$ Tagliamento $\left.{ }^{36}\right)$. L'objet exposé, bricolé, rafistolé, est par nécessité de la pire espèce : on a pris ce qu'on avait « sous la main »...

\section{Acteur / objet}

Fig.7.

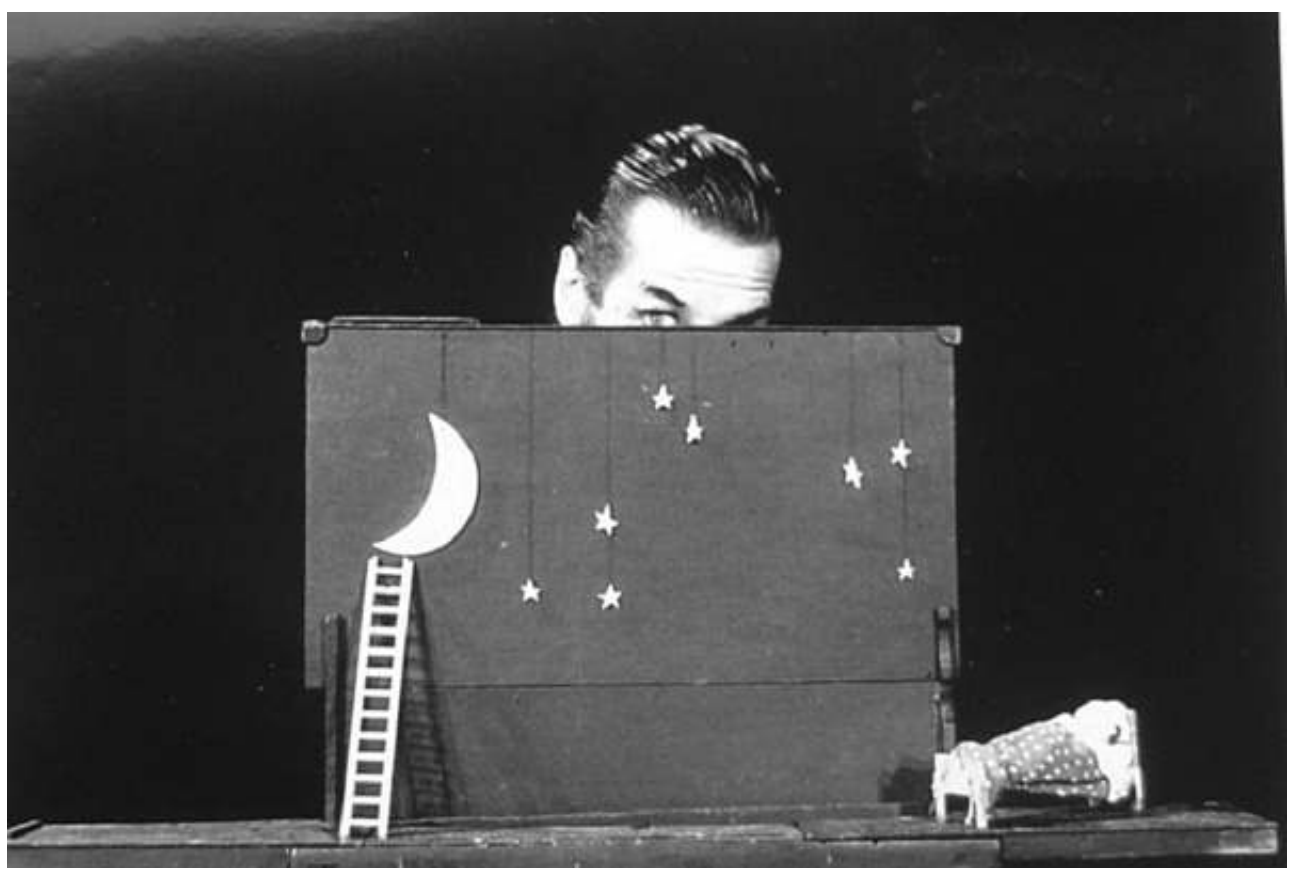

Petit plateau, petite jauge : le conte est aussi rendu possible par les nouvelles dimensions de l'espace théâtral. Théâtre Manarf, Paris Bonjour. Création en 1979 à la MJC d'Avrilié.

Cliché : R. Sauloup

Ce type d'objet impose donc à l'acteur un jeu particulier. L'objet par exemple permet de raconter une multitude d'histoires (il suffit pour cela d'accrocher son imagination à quelqu'une de ses éraflures), qui transforment le comédien en ce conteur qui avait presque disparu de nos sociétés, d'autant que le plateau de jeu aux dimensions réduites souvent à celles d'une table de cuisine induit en outre un rapprochement avec le spectateur (Fig. 7), et que le conte suppose justement une adresse particulière au 
public, un ménagement constant, par le regard et le ton, du lien vivant entre l'acteur et l'auditoire, comme dans le théâtre de rue. L'objet favorise donc la parole, dans une forme de théatre qui n'a souvent aucunement recours au texte littéraire. Il donne également lieu à un jeu plus distancié, moins psychologique et moins illusionniste. Comme le dit Christian Carrignon, ce sont alors deux spectacles que l'on montre en un seul: celui de la fiction et celui du mécanisme à l'oeuvre pour sa fabrication. Le comédien y devient un "actant ", c'est-à-dire un être de chair dont les places et les fonctions varient rapidement au cours du spectacle, et qui est très souvent, par exemple, comédien en même temps que machiniste. Dans une séquence de Catalogue de voyage, une valise remplie de sable, ouverte face aux spectateurs, contient un petit camion rouge que le comédien fait rouler : il imite le bruit du moteur avec ses lèvres, comme un enfant. Mais, tout en manipulant ce jouet, Christian Carrignon incarne aussi le conducteur, accoudé à sa portière, gagné par la fatigue. Il joue donc le rôle du camionneur en même temps qu'il déplace le camion, extrait du sable un nouvel objet, manifeste les émotions, et met en marche la musique de la fin.

Fig.8.

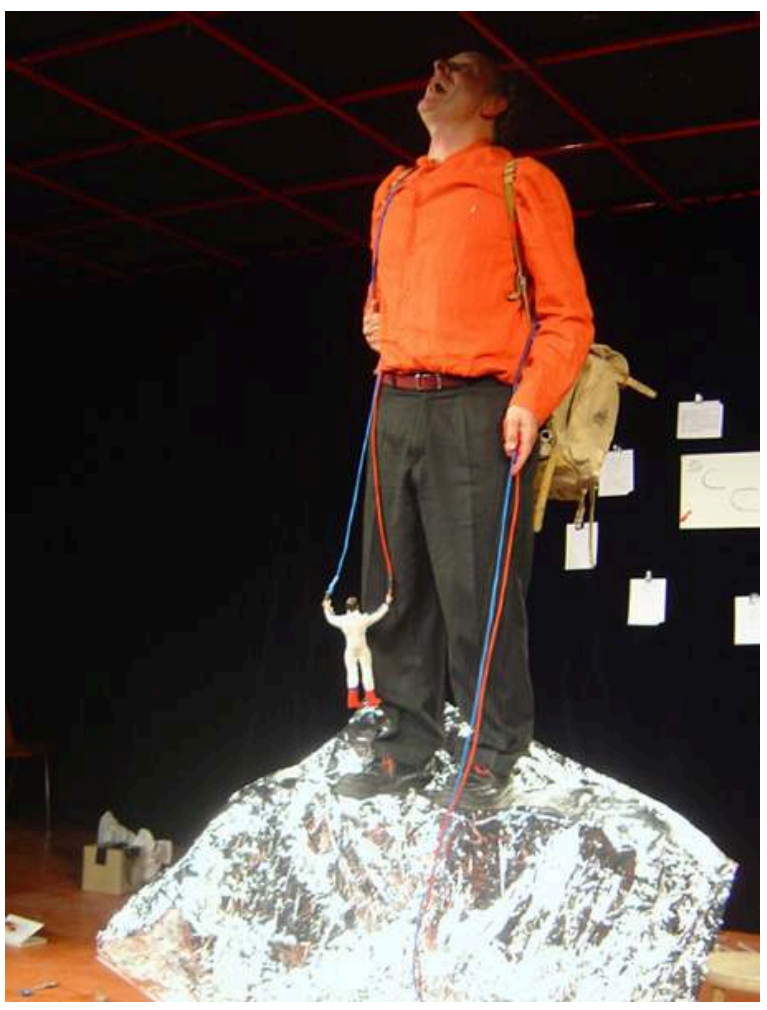

La figurine n'est pas animée ; elle est plutôt ballottée au gré des mouvements du comédien. Théâtre de Cuisine, Catalogue de voyage. Création en octobre 1981 au Centre de Recherche de Pau.

Cliché : J.-L. Mattéoli

31 L'objet pauvre produit ainsi une révision de la hiérarchie traditionnelle entre l'acteur et l'objet. Dans certaines formes de théâtre d'objet, il a par exemple conduit, selon Roland Shön, à donner à l'acteur « une place autre », hybride, comme on vient de le voir : une sorte de « découronnement » de l'acteur par l'objet se produit, mais qui ne conduit pas pour autant à une éviction totale. Car le paradoxe du théâtre d'objet est que jamais l'acteur n'est davantage présent, puisqu'il assure souvent à lui seul le jeu et la machinerie! Le comédien de surcroît ne fait plus preuve d'aucune technique 
particulière pour manipuler l'objet, contrairement aux soins apportés par le marionnettiste qui entoure sa créature de bois ou de chiffons pour l'animer. Comme l'écrit Christian Carrignon, l'apparition de l'objet dans le champ des pratiques marionnettiques s'est effectué «par glissement progressif, vers moins de naturalisme, moins de technique, moins de personnification, plus d'abstraction... $»^{37}$ (Fig. 8). L'objet est simplement exposé à la vue des spectateurs, parfois déplacé comme le feraient les enfants, sans dextérité ou technique particulière. Dans Grigris $^{38}$, Roland Shön se contente de se placer autour des spectateurs en évoquant l'objet que ceux-ci continuent de regarder sur la scène, l'animation étant en quelque sorte immobile.

Dans les spectacles du Radeau, le comédien, également acteur et machiniste, se livre à une lutte continuelle avec les objets qui, tous, entravant son jeu comme ses déplacements, finissent par conférer une sorte de dignité et de grâce à sa piètre humanité. Veut-il franchir les tables de formica installées parallèlement au bord de la scène occupant tout l'espace qu'il est obligé à d'inélégantes contorsions; les multiples chaises à piètement métallique dont il s'encombre parfois provoquent des bruits qui couvrent le texte qu'il prononce; les costumes mal boutonnés bâillent ridiculement dès qu'un dos s'offre aux regards ; les maquillages grossièrement passés à vue se craquèlent ou ruissellent sous la chaleur des éclairages ; les lourdes lunettes d'écaille, la robe rose passée par-dessus un costume de ville, en bref tous les objets tendent à défaire de son prestige un acteur qu'une opinion commune nous peint comme le prince de la scène. Mais ce rang n'est pas dû au decor(um) qui l'entoure ; c'est parce qu'il parle qu'il est grand, et que sa parole, porteuse des textes fondateurs de la littérature philosophique, historique, poétique et théâtrale, l'emporte sur cet espace dévasté, rétif à la métaphorisation, parfaitement ingrat, d'où toute illusion est bannie, mais où le théâtre est retrouvé. 
Fig.9.

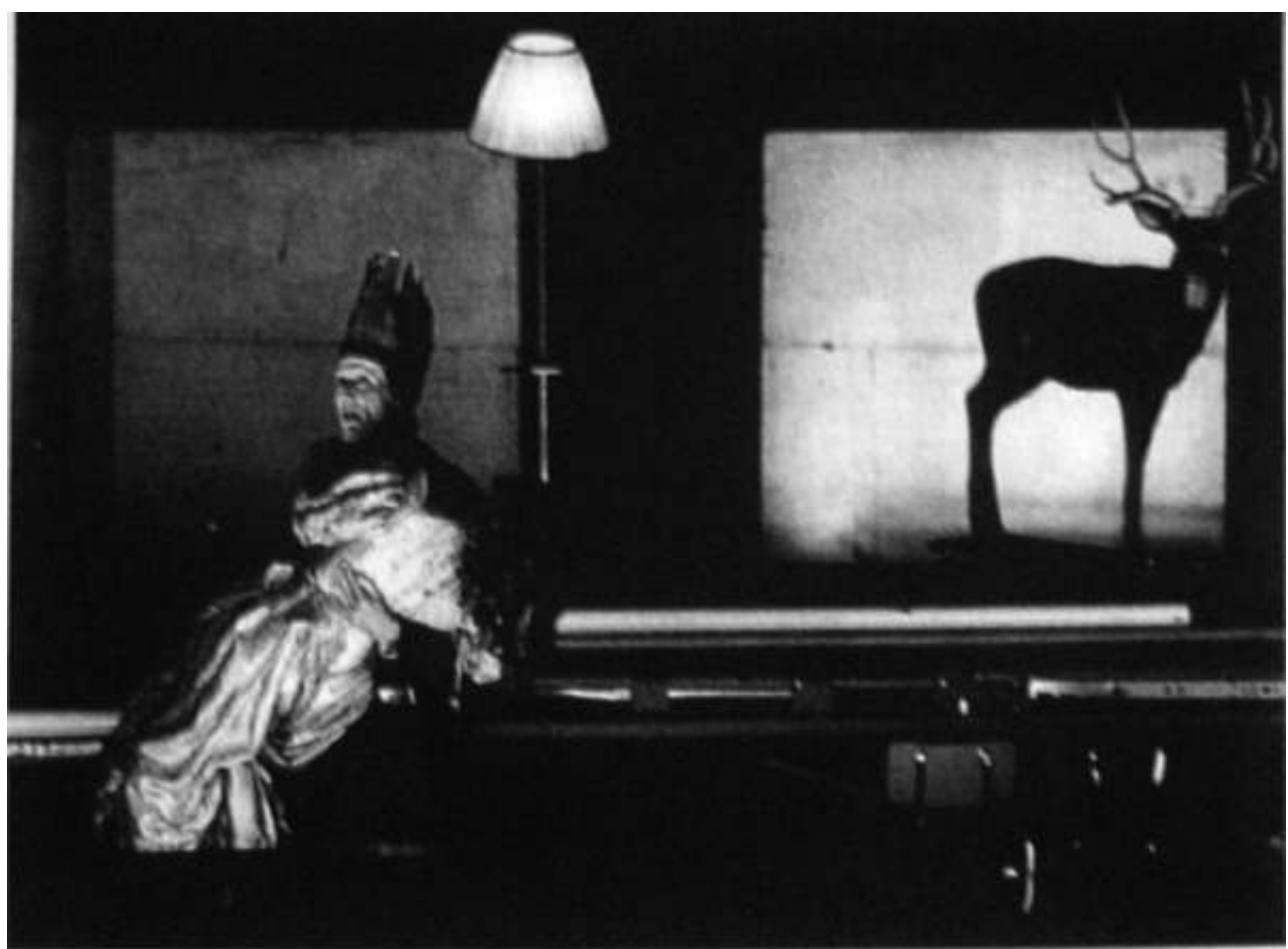

Le cerf en plastique exposé en fond de scène parasite les textes, en un trait d'humour actualisable à volonté. Théâtre du Radeau, Orphéon. Création en novembre 1998 au Théâtre national de Bretagne.

Cliché : A. Dugas

Ici aussi l'objet s'expose, concourant à faire naitre des moments d'humour diversement perçu par les spectateurs. Ainsi, le cerf de plastique (Fig. 9), demeure en fond de scène pendant une grande partie d' Orphéon ${ }^{39}$. Jamais pris en compte par les acteurs, détaché de tout rapport évident avec le texte ou les actions auxquelles le spectateur assiste, sa présence finit par être oubliée, comme absentée du champ de vision. Puis il réapparaît, soudain pertinent, en une fulgurance humoristique dont le siège est peut-être dans l'esprit du seul spectateur (il est rare en effet que des rires saisissent de manière unanime une salle du Radeau). Le cerf de plastique vert est disponible, "prêt ", en même temps qu'il refuse obstinément de « servir » quelque dessein dramaturgique que ce soit : il contribue plutôt à l'ouverture toujours recommencée du sens, à l'évitement de sa fossilisation en significations ou narrations, comme les motifs de Bach ou l'écriture de Kafka dont François Tanguy est familier. « Une dynamique [...] se substitue à l'objet du désir et n'est jamais atteint. Ça donne lieu à des figures incompossibles, qui ne peuvent totalement recouvrir ce que ça voudrait décrire ", écrit-il ${ }^{40}$.

\section{Retour à l'histoire}

Il reste maintenant à s'interroger sur les possibles congruences entre le phénomène de dé-métaphorisation que nous venons d'observer et la période, du milieu des années 1970 à aujourd'hui, où il se développe. Une première explication à ce déficit de manipulation et de métaphorisation peut être cherchée du côté du contexte historique stricto sensu. L'objet pauvre apparaît à peu près simultanément dans plusieurs endroits du théâtre au milieu des années 1970 : c'est là une période de désenchantement et de 
souvenir des mouvements sociaux de 1968, marquée par la fin des espoirs de changement et l'avènement progressif d'une autre période, moins perméable à l'utopie ou aux interrogations, plus ouverte à la rentabilité. En même temps, les formes et les pratiques de l'action nées en 1968 («Ne laissons personne parler à notre place ») continuent à inspirer ceux qui inventent le théâtre dans ces années-là ${ }^{41}$. C'est d'ailleurs dans la foulée des événements de 1968 que le terme "manipulation ", s'appliquant à la politique et à l'idéologie, se répand ${ }^{42}$, traduisant la méfiance qu'éprouve la masse des gouvernés par rapport aux discours institutionnels. C'est dans ce contexte que s'introduisent, à la marge, les objets réels, ordinaires et pauvres, réfutant l'illusion traditionnelle, perçue désormais comme une forme de prestidigitation aux limites de la malhonnêteté: Barbie, Ken, ou les figurines Playmobil, demeurent difficilement réductibles à une autre identité que la leur, et rarement le comédien cherche à donner l'illusion qu'ils sont vivants.

Dans « manipulation », au-delà de l'opération marionnettique qui consiste à donner à un objet l'apparence du mouvement autonome et de la pensée, il y a donc toujours les sens négatifs de « manœuvre occulte, persuasion pour amener quelqu'un à agir comme on le souhaite ", ou de "prestidigitation ». Et si Christian Carrignon tient à distinguer le théâtre d'objet du théâtre de marionnettes (comme le font également Roland Shön ou Michel Laubu, le directeur du Théâtre Turak $\left.{ }^{43}\right)$, c'est que quelque chose de fondamental les sépare à ses yeux : la fabrication ou la transformation de l'objet pour les besoins du spectacle, c'est-à-dire bien sûr sa manipulation une fois en scène (celle-ci peut toujours se produire) mais aussi sa manipulation avant, pour que l'objet, qui, je le rappelle, a une vie et une voix, ne soit pas contraint par plus puissant que lui à dire ou faire ce à quoi sa singularité se refuse. Michel Laubu proclame que l'objet "parle », qu'il "propose des choses $»^{44}$. Être à son écoute dans ce dialogue, c'est donc user, par exemple, davantage de la métonymie que de la métaphore ("le camion est un lieu»). Résultat : en même temps qu'une forme d'égalité se constitue entre ces partenaires que sont l'objet et l'acteur, leurs fonctions dans le spectacle subissent des déplacements significatifs.

Une autre explication peut être avancée à ce déficit de manipulation et de métaphorisation, cette fois d'ordre artistique. Les arts plastiques, qui interrogent aussi l'objet pauvre depuis le début du XX $\mathrm{XX}^{\mathrm{e}}$ siècle, exercent en effet leur influence sur le milieu du théâtre d'objet - d'autant plus que l'origine des créateurs est souvent plasticienne: «Jacques Templeraud et moi venons tous les deux des Beaux-Arts, où, sans le savoir, nous pratiquions déjà ce genre d'expression. Il nous était plus facile de montrer directement aux gens ce que nous bricolions que de passer par le système des expositions", raconte Charlot Lemoine à propos des débuts du théâtre d'objet ${ }^{45}$. Historiquement en effet, le rôle actif dévolu au matériau de départ, qui peut aussi être un objet trouvé, est le fait de plasticiens. Kurt Schwitters déclarait qu'il convenait d'user du mauvais matériau et du mauvais outil pour parvenir à un bon résultat; et Jean Dubuffet écrit, un demi-siècle plus tard, que «l'homme doit parler, mais l'outil aussi et le matériau aussi $»^{46}$. Ainsi, c'est du côté des arts plastiques que se placent, avec des nuances diverses, les créateurs du théâtre d'objet. Christian Carrignon y situe l'une des origines du théâtre d'objet (ready-made de Marcel Duchamp, collages dada, etc.). Roland Shön, lui, dit se considérer, dans ses spectacles, comme un «transmetteur de l'art inventé au siècle dernier $\aleph^{47}$, notamment collages et assemblages. Pour Michel 
Laubu, enfin, le théâtre d'objet est « au carrefour des arts plastiques, de la musique, de la danse, du théâtre et des sciences physiques quelquefois $»^{48}$.

Fig. 10.

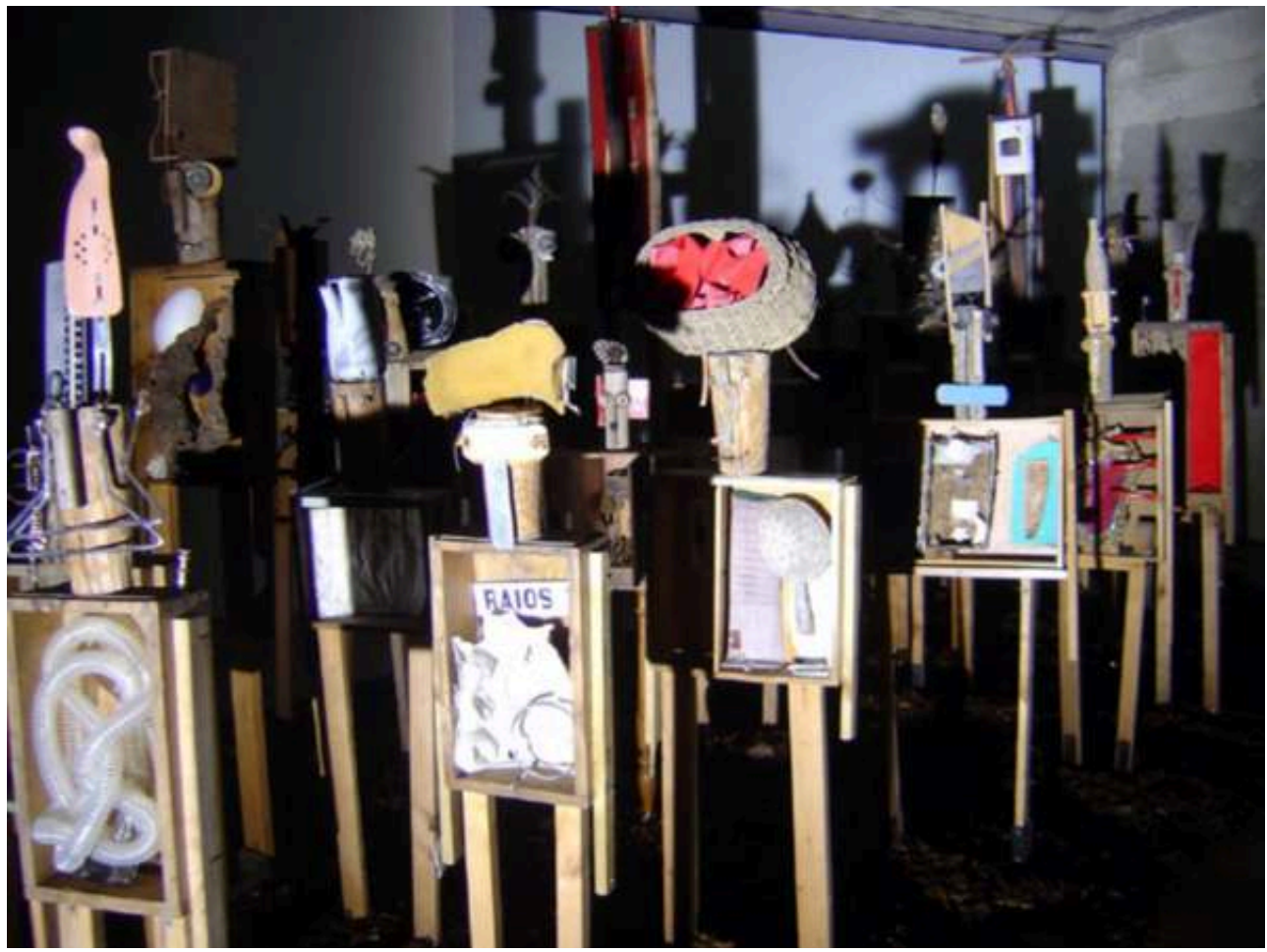

Antonio Catalano, Univers sensibles. Création en juin 2005 à la BIAM de La Villette.

cliché : J.-L. Mattéoli 
Fig. 11 .

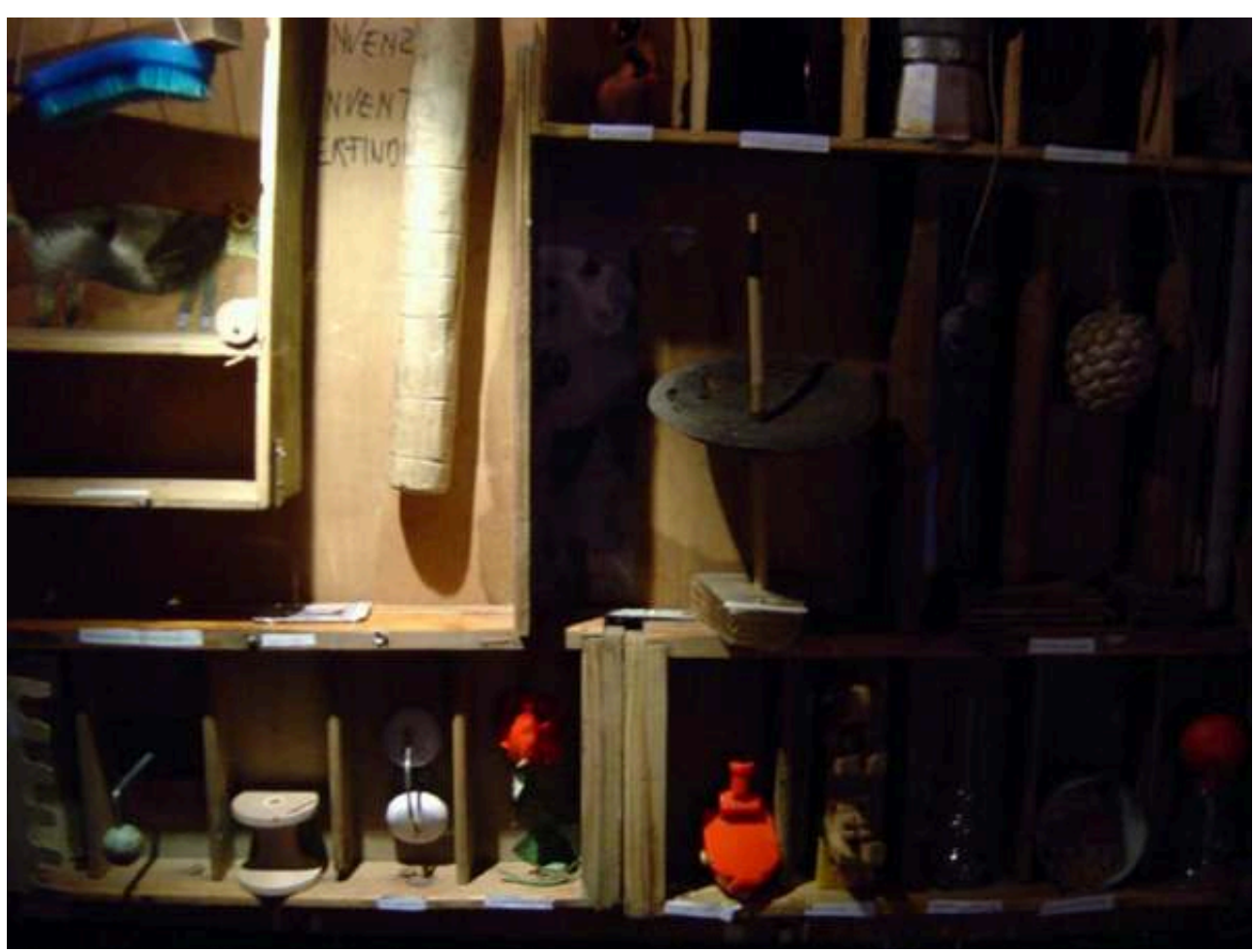

Le spectacle prend l'allure d'une exposition, composée de plusieurs « stations » entre lesquelles les spectateurs évoluent.. Antonio Catalano, Univers sensibles. Création en juin 2005 à la BIAM de La Villette.

Cliché : J.-L. Mattéoli

En outre, l'objet pauvre a semble-t-il imposé aujourd'hui une sorte de logique thématique à ceux qui en usent dans leur théâtre et aux histoires qu'ils inventent : la collection (particulière ou ethnographique), l'exposition (en série, ou individuelle, artistique ou scientifique), le cabinet de curiosités de personnages imaginaires sont devenus des constantes des histoires racontées et des installations scéniques, générant par là des conduites particulières de la part du public, plus proches de la fréquentation d'une exposition ou d'un musée (par exemple de déambulation autour d'objets, ethnographiques ou artistiques). De même, il est fréquent d'observer la présence d'une exposition parallèlement à un spectacle, soit de manière indépendante, soit en relation avec lui. Pour Bestiaire forain, le Théâtre de La Licorne propose ainsi une exposition d'animaux-machines avant la représentation, sous couvert de ménagerie. Il peut arriver aussi, phénomène ultime et très révélateur dans le renversement qui l'exprime, $\mathrm{qu}^{\prime}$ « un spectacle [soit] proposé au coeur de l'exposition». C'est le cas pour Univers sensibles d'Antonio Catalano, présenté lors de la Biennale internationale des arts de la marionnette en juin $2005^{49}$. Dans le travail de ce metteur en scène italien, l'objet est rarement manipulé (ou a minima), et n'est jamais marionnettisé. Il n'y a en effet, dans les différents moments de spectacle ou de déambulation que cet artiste propose, aucune animation d'objet par les comédiens. Certains lieux, comme « Le Pavillon des merveilles du magicien Cotrone ", «Le Pavillon des grands rêveurs » ou " Le Peuple de l'automne » (Fig. 10 et 11), ressemblent plutôt à une installation plastique dans laquelle le public est tenu à distance des objets par le discours des guides qui ne les manipulent pas non plus, mais en racontent l'histoire. 

faire, apparemment, avec les principes d'étrangéisation du théâtre brechtien qu'avec l'incarnation du théâtre aristotélicien. L'illusion a donc, dans certains cas, cédé le pas à la monstration, la prestidigitation (toujours suspecte de manipulation) à la déhiérarchisation, le mouvement à l'immobilité. Même s'il n'est pas général, ce glissement affecte cependant de manière visible les travaux des compagnies qui oeuvrent avec l'objet pauvre (même dans les arts de la rue). En effet, avec ce type d'objet extrait du monde réel, c'est comme si une certaine dose de réalité parasitait ce lieu traditionnel de l'illusion, à laquelle l'accessoire apportait hier sa contribution. Dans les spectacles du Théâtre du Radeau par exemple (dont le travail constitue par ailleurs comme une limite de l'entreprise d'appauvrissement), objets, costumes et lumières affirment la scène comme un lieu de réalité. Le corps réel de l'acteur, le poids ou le bruit réels de l'objet, la source réelle de lumière, le tout dans la réalité d'un espace partagé de plain pied par les spectateurs : c'est une certaine « magie » de l'événement théâtral qui est de la sorte rejetée. Ainsi s'affirme un théâtre de la distance, à l'opposé de toute prestidigitation et au service de l'aiguisement de la perception, réalisant une synthèse inédite entre Brecht et Artaud. L'objet, dans ce cas, est un crible qui retient, dans un espace scénique dégradé, toute extrapolation des significations et affirme, dans sa matérialité déplacée (qu'elle soit obstinément décorative ou fonctionnelle), le caractère concret de la scène, au rebours de tout espace d'illusion et d'envoûtement. La quotidienneté y domine en outre la gestuelle des acteurs, dans leur relation aux objets de ce théâtre soucieux de faire parler à la scène "son langage ", comme le proposait Antonin Artaud dès 1933.

L'objet pauvre, qu'il soit récupéré (et donc marqué par son usage passé) ou anonyme (à force d'avoir trop partagé notre quotidien), appelle la prise de parole davantage, peutêtre, que la prise en mains. Massimo Schuster déclare : «Pour donner à la marionnette tout son pouvoir d'expression, je la manipule le moins possible. C'est pour moi le moyen d'aller à l'essentiel $\aleph^{50}$. Comme si les grandes marionnettes fabriquées par Enrico Baj étaient ni plus ni moins que des objets ordinaires, résistant comme eux à la manipulation traditionnelle. C'est que dans le fond, un certain travail théâtral s'effectue aujourd'hui dans le contact d'objets réels, porteurs de mémoire, bricolés par des hommes, exposés sur la scène : ils y apportent, à leur manière, cette lucidité (la distance) traversée d'ombres (la mémoire) qui est la leçon en demi-teintes du dernier tiers du XXe siècle.

\section{NOTES}

1. Tadeusz KANTOR, «Entretien avec Philippe du Vignal (1985), in Entretiens, Paris, Éditions Carré, coll. Art \& esthétique, 1996, p. 75. Dans son entretien avec Denis Bablet en 1972, Kantor infirme l'idée selon laquelle il aurait commencé par la peinture pour aller vers le théâtre : «J'ai travaillé parallèlement dans les domaines de la peinture et du théâtre [...] dès le début. Je pourrais dire dès mes études. A l'Académie, j'étudiais la peinture, mais aussi la scénographie avec

Images Re-vues, 4 | 2007 
Karol Frycz, l'ami de Gordon Craig » (T. KANTOR, «Entretien avec Denis Bablet » (1972), in Entretiens, op. cit. , p. 25).

2. Tadeusz KANTOR, Leçons de Milan, traduit du polonais par Marie-Thérèse Vido-Rzewuska, Arles, Actes Sud-Papiers, 1990, p. 18.

3. Id., p. 81.

4. Pierre BERGOUNIOUX, Back in the sixties, Lagrasse, Verdier, 2003, p. 21.

5. C'est là le titre d'un article consacré par Michel Deutsch aux pratiques théâtrales qui s'écartent à la fois de «la scène somptueusement décorée " ou de "la scène vide (la scène de l'acteur et du texte) », les deux pratiques dominantes du théâtre régulier (Michel DEUTSCH, « Dans la marge », in Le Théâtre et l'Air du temps, Paris, L'Arche, 1999, p. 15).

6. André ANTOINE, «Le Théâtre-Libre » (1890), in Jean-Pierre SARRAZAC et Philippe MARCEROU, Antoine, l'invention de la mise en scène, anthologie des textes d'André Antoine, Arles, Actes SudPapiers / Centre national du théâtre, 1999, p. 79.

7. Jerzy GROTOWSKI, «Vers un théâtre pauvre » (1965), in Vers un théâtre pauvre, traduit du polonais par Claude B. Levenson, Lausanne, L'Age d'Homme, 1968, p. 20.

8. Louis JOUVET, "Problèmes du théâtre ", in Réflexions du comédien, 1940, Paris, Librairie Théâtrale, 1986, p. 168-172.

9. Spectacle de la compagnie Deschamps \& Makeïeff, création au Théâtre de Nîmes en 2003.

10. Vsevolod MEYERHOLD, « Histoire et technique du théâtre ", 1907, in Écrits sur le théâtre 1, traduit du russe par Béatrice Picon-Vallin, Lausanne, L'Age d'Homme, 1973, rééd. 2001, p. 92. Cette formule, citée par Meyerhold dans son étude, est de Schopenhauer.

11. Id., p. 96.

12. «Lorsqu'elle se limite au strict nécessaire [...], l'architecture scénique semble parfois parcimonieuse. Elle fait "pauvre" » (Bertolt BRECHT, « Sur l'architecture scénique », in Écrits sur le théâtre 1, traduit de l'allemand par Jean Tailleur, Guy Delfel, Béatrice Perregaux et Jean Jourdheuil, Paris, L'Arche, 1972, p. 435). Il est évident que cette pauvreté ne s'entend que par rapport à la « richesse » des décors exacts du naturalisme d'Antoine ou de Stanislavski.

13. Tadeusz KANTOR, «Entretien avec Denis Bablet» (1972), loc.cit., p. 41 ; je souligne.

14. Tadeusz KANTOR, Leçons de Milan, traduit du polonais par Marie-Thérèse Vido-Rzewuska, Arles, Actes sud, 1990, p. 19.

15. Quels éléments choisir pour installer sur scène cette " reproduction pleine de lacunes » qui doit faire place à "l'illusion intégrale »? En usant par exemple «d'objets bien épais, réalistes, d'occasion si l'on veut ("second hand"). Un bâti de porte, par exemple, pris sur un chantier de démolition, arraché à un mur, un objet qui a une expérience sociale et une biographie, et non une construction abstraite, signifiant un passage et dépourvue de toute autre propriété que celle précisément de permettre aux gens de passer » (Bertolt BRECHT, «Sur l'architecture scénique », op. cit., p. 440).

16. Tadeusz KANTOR, Leçons de Milan, op. cit., p. 19 ; je souligne.

17. Tadeusz KANTOR, « Le Théâtre indépendant, 1942-1944 », in Le Théâtre de la mort, op. cit, p. 32 ; c'est l'auteur qui souligne. Plus tard, Kantor déclare : « Pour moi, si je construis les émotions, c'est parce que quelqu'un doit pleurer » («Entretien avec Anne Ubesfeld», loc. cit., p. 57).

18. Kurt SCHWITTERS, " [Texte autobiographique]», in Merz, écrits choisis et présentés par Marc DACHY, traduits de l'allemand par M. Dachy et C. Graber, de l'anglais par M. Dachy, Paris, Éditions Gérard Lebovici, 1990, p. 169. Pour Tadeusz Kantor, l'expérience s'effectue en des termes similaires : «Nous n'avions assez de force que pour attraper / CE QUI ÉTAIT SOUS LA MAIN, L'OBJET RÉEL / et le proclamer oeuvre d'art! » (Tadeusz KANTOR, Leçons de Milan, op. cit., p. 19). 19. "J'y suis souvent, [au marché aux Puces de Saint-Ouen], en quête de ces objets qu'on ne trouve nulle part ailleurs, démodés, fragmentés, inutilisables, presque incompréhensibles...» (André BRETON, Nadja (1924), Paris, Gallimard, coll. Folio, 1975, p. 62). 
20. Anne UBERSFELD, "L'Objet théâtral contemporain », Actualités des arts plastiques $n^{\circ} 40$ (« L'Objet théâtral »), Paris, CNDP, 1980, rééd. 1984, p. VII.

21. Plasticien, constructeur et patineur d'objets pour des compagnies de théâtre de rue, Nicolas Diaz vit et crée à Paris.

22. Antoine PROST, Douze leçons sur l'histoire, Paris, Le Seuil, coll. Points, 1996, p. 298. L'expression « basculement mémoriel des années 1970 » se trouve p. 299.

23. Georges BANU, "De l'histoire vers la mémoire... », in Les Cahiers de l'Herne («Opéra, théâtre, une mémoire imaginaire »), Paris, 1990, p. 11.

24. Pierre BLAISE, «Un "Théâtre d'art" de marionnettistes », in Carnets de la marionnette (« Les Fondamentaux de la manipulation »), Paris, THEMAA / Éditions Théâtrales, 2003, p. 57.

25. Christian Carrignon bâtit un spectacle, La Conférence des petits papiers, sur un bien pauvre matériau, les papiers que, dans une longue promenade de trois jours dans une ville, il ramasse dans les caniveaux ou sur les trottoirs, déchirés, froissés, abandonnés : « Quand je trouve un joli papier, je m'invente l'avant et l'après, une histoire [...]. Comment procède un archéologue, un paléontologue?» (C. CARRIGNON, La Conférence des petits papiers, tract de présentation, Marseille).

26. Emmanuel HOCQUART, «La Bibliothèque de Trieste » (1987), in Ma haie , Paris, P.O.L, 2001, p. 26.

27. Id ., « Entretien avec Stéphane Baquey » (1995), in Ma haie, op.cit., p. 279.

28. Id ., p. 289. "Il a suffi de copier », écrit encore Emmanuel Hocquart, « Copier, c'est gommer. Quelle est mon intention? Je dirais une intention de pauvreté » (" Ma vie privée » (1995), in Ma haie, op. cit., p. 272).

29. Spectacle du Théâtre de Cuisine, création au centre de Recherche de Pau en octobre 1981.

30. Co-directeur du Théâtre de Cuisine avec Katy Deville, Christian Carrignon habite et crée à Marseille. La compagnie existe depuis 1979. «L'objet », écrit-il, "n'est définitivement plus une marionnette, c'est un "lieu" ».

31. Spectacle du Théâtre de Cuisine, création en mars 1982 au Théâtre de Cergy-Pontoise.

32. À propos de cette différence entre marionnette réaliste et marionnette fruste, la seconde étant la plus fidèle à l'essence du phénomène marionnettique, voir « L'Âme et "la partie pour le tout" ", in Antoine VITEZ, Le Théâtre des idées , anthologie proposée par Danièle Sallenave et Georges Banu, Paris, Gallimard, coll. « Le Messager », 1991, p. 263-264.

33. Claude LÉVI-STRAUSS, La Pensée sauvage (1962), Plon, Agora Pocket, 1990, p. 33.

34. Spectacle du Théâtre de La Licorne, création en novembre 2001 au Channel, Scène nationale de Calais.

35. Spectacle de la compagnie Deschamps \& Makeïeff, création au Théâtre de Nîmes le 16 mai 1994.

36. Spectacle du Théâtre du Radeau, mise en scène de François Tanguy. Création en juillet 1996 au Kuntzfest de Weimar.

37. Christian CARRIGNON, « Théâtre d'objet : mode d'emploi », Marseille, mars 2000, non publié.

38. Spectacle du Théâtrenciel, mise en scène Roland Shön, création en 1992 à Dieppe.

39. Spectacle du Théâtre du Radeau, mise en scène de François Tanguy. Création en 1998 à St Jacques de la Lande pour le Théâtre national de Bretagne.

40. François TANGUY, entretien, Le Mans, 17 juin 2005.

41. Le slogan de 1968 date du 24 mai (cité in Kristin ROSS, Mai 28 et ses vies ultérieures, 2002, traduit de l'anglais (américain) par Anne-Laure Vignaux, Paris, éditions Complexe / Le Monde diplomatique, 2005, p. 77) ; quant à la manière dont le théâtre de ces années-là y souscrit, il faudrait évoquer en sus des compagnies dont nous parlons, toute la mouvance du Théâtre du quotidien à partir de 1974 à Théâtre ouvert, les installations de quatre compagnies de théâtre à la Cartoucherie de Vincennes entre 1970 et 1973, les mutations du cirque et de la rue, etc. 
42. Alain REY (dir.), Dictionnaire étymologique de la langue française, Paris, Le Robert, 1992, p. 1183a.

43. « Le théâtre d'objet est l'enfant rebelle de la marionnette, il donne des coups de pied dans les fils » (Michel LAUBU, programme de Critures, l'épopée ordinaire, spectacle créé en 1995 à Lyon). Dans un autre texte («C'est quoi le théâtre d'objet?») Michel Laubu introduit ce sous-titre : « Pourquoi je n'utilise pas le terme de "marionnette" pour désigner mon travail ».

44. Michel LAUBU, directeur du Turak Théâtre (Lyon), entretien, 27 janvier 2005.

45. Charlot LEMOINE (directeur du Vélo Théâtre), entretien avec Roger Wallet, Marionnettes $\mathrm{n}^{\circ}$ 7, Charleville-Mézières, 1985, p. 46. Jacques Templeraud est le directeur du Théâtre Manarf.

46. Jean DUBUFFET, « Notes pour les fins-lettrés», in Prospectus et tous écrits suivants (1973), Paris, Gallimard, Coll. Folio Essais, 1995, p. 23.

47. Roland SHÖN, entretien, 7 avril 2005.

48. Michel LAUBU, «C'est quoi le théâtre d'objet? ", programme du Théâtre de la Croix-Rousse, Lyon, 15 au 28 mars 2002.

49. Univers sensibles, spectacle d'Antonio Catalano, rassemblement de divers spectacles présentés sous ce titre depuis 2001, création à la Biennale internationale des arts de la marionnette à La Villette, mai 2005. La formule figure dans le livret de présentation de la Biennale (je souligne).

50. Stéphanie TESSON, « Le Mahabharata de Massimo Schuster », L'Avant-Scène Théâtr , $\mathrm{n}^{\circ}$ 1155, janvier 2004, p. 19.

\section{RÉSUMÉS}

Afin de comprendre les qualités et vertus de l'objet "pauvre » dans le théâtre contemporain, il est nécessaire de revenir à Thadeusz Kantor et sur l'usage qu'il prônait d'un objet arraché au réel, condition même pour l'acteur d'être sur scène. Le pouvoir mnésique des objets qui échouent sur scène contribue notamment à rapprocher le théâtre des pratiques plastiques. Littéralement invisibles, indices métonymiques d'un ensemble ou plus directement métaphoriques, nous envisagerons leurs différents modes d'insertion scénique. Ils marquent en effet les diverses critiques et alternatives du naturalisme dans le théatre moderne, de Meyerhold à Grotowski (artisan du "théâtre pauvre ») en passant par Brecht. L'objet intervient non sans lacune et mystère (l'objet trouvé cher à André Breton) à l'égard d'un monde qui l'a rejeté en fragment de ses épisodes traumatiques. Par quel retournement de l'objet sur l'histoire, depuis les années 1970, s'est produit la désaffection pour la métaphore, encouragée dans le théâtre contemporain (Théâtre de Cuisine, Théâtre de la Licorne, Théâtre du Radeau...) par une relation à l'objet devenue « bricolage »?

\section{INDEX}

Mots-clés : accessoire, arts plastiques, mémoire, métonymie, récupération, réel

Thèmes : théâtre

Index géographique : France

Index chronologique : XXe siècle 


\section{AUTEUR}

\section{JEAN-LUC MATTÉOLI}

Jean-Luc Mattéoli est titulaire d'une thèse de doctorat : " L'objet pauvre, mémoire et quotidien sur les scènes contemporaines françaises ». Il occupe un poste à l'IUFM de Bourgogne et enseigne à l'Université de Dijon et à Paris III

jl.matteoli@gmail.com 